\title{
Additions to the orchid flora of Peninsular India
}

\author{
Kaliamoorthy S. \& T.S. Saravanan \\ National Orchidarium \& Experimental Garden, Botanical Survey of India, \\ Southern Regional Centre, Yercaud, Salem District, Tamil Nadu - 636 602, India. \\ E-mail: bsinoegyercaud@gmail.com
}

\begin{abstract}
Oberonia maxima C.S.P.Parish ex Hook.f., and Thrixspermum formosanum (Hayata) Schltr., so far known from northeast India, are reported here for the first time from Tamil Nadu, Peninsular India. Detailed descriptions and photographs are provided for easy identification.
\end{abstract}

Keywords: New Record, Oberonia maxima, Orchidaceae, Tamil Nadu, Thrixspermum formosanum.

\section{Introduction}

During recent field explorations in the forests of Mukurthi National Park, Nilgiri Biosphere Reserve, Tamil Nadu, the authors have collected interesting specimens belonging to the genera Oberonia and Thrixspermum of Orchidaceae. The genus Oberonia Lindl. is represented by about 350 species (Prasad et al., 2018; Govaerts et al., 2019) while Thrixspermum Lour by c. 151 species (The Plant List, 2013). Sixty nine species of Oberonia have been reported from India, mainly found in Peninsular India, north-eastern states, Andaman and Nicobar Islands, central India and Western Himalaya (Ansari \& Balakrishnan, 1990; Kumar \& Manilal, 1994; Misra, 2007; Narayanan et al., 2010; Chowlu et al., 2015; Chowlu \& Rab, 2017; Prasad et al., 2018). Thrixspermum is represented by about 16 species in India (Misra, 2007; Maina et al., 1998; Mao et al., 2011; Gogoi, 2011; Gogoi \& Yonzone, 2015), of which 4 are reported from Peninsular India (Misra, 2007).

Critical examination of the collected specimens and perusal of the relevant literature (Deori \& Hajra,

Received: 27.06.2019; Revised \& Accepted: 18.12.2019

Published Online: 31.12.2019
1975; Liu \& Su, 1978; Ansari \& Balakrishnan, 1990; Hynniewta et al., 2000; Kumar \& Kumar, 2005; Chen \& Wood, 2009; Gogoi \& Yonzone, 2015; Geiger, 2016) revealed their identity as Oberonia maxima C.S.P.Parish ex Hook.f. and Thrixspermum formosanum (Hayata) Schltr. respectively. They are reported here as new additions to the flora of Peninsular India.

Oberonia maxima C.S.P.Parish ex Hook.f., Fl. Brit. India 5: 677. 1888; Deb, Bull. Bot. Surv. India 3(2): 128. 1961; Seidenf., Dansk Bot. Ark. 25(3): 21. 1968; Deori \& Hajra, Bull. Bot. Surv. India 17(1-4): 170. 1975; Seidenf., Dansk Bot. Ark. 33(1): 13. 1978; Jain \& Mehrotra, Prel. Inv. Orch. India 97. 1984; R.Ansari \& N.P.Balak., Orch. Monogr. 4: 22. f. 16. 1990; Hynn. et al. in Hajra \& Chatterjee, Orch. Nagaland 224. 2000; A.S.Chauhan in P. Pathak et al., Orch. Sci. \& Com. 93. 2001; C.S.Kumar \& P.C.S.Kumar, Rheedea 15(1): 50. 2005; D.L.Geiger, Orchids 85(5): 390. 2016. Type: BURMA, Moulmein, 1869, Parish C.S.P. 287 (holo K000387694 digital image) Fig. 1a \& b

Epiphytic, pendulous, caespitose herbs. Roots many, pale brownish-white, $2.4-10.5 \mathrm{~cm}$ long. Leaves 4-6, pale green, fleshy, distichous, 7.5-15.6 $\times 1.1-1.6 \mathrm{~cm}$, ensiform, articulate at base, acute at apex, entire. Inflorescence terminal, longer than the leaves, densely many flowered, decurved, compressed and adnate to the reduced uppermost leaf, c. $16.9 \mathrm{~cm}$ long, scape $c .4 .2 \mathrm{~cm}$ long, rachis $c$. $12.7 \mathrm{~cm}$ long. Flowers c. $3.5 \mathrm{~mm}$ long, greenishyellow, sub-sessile or with a short pedicel, closely adpressed to the rachis; floral bracts longer than the pedicel plus ovary, c. $2.3 \times 1.2 \mathrm{~mm}$, oblong, 

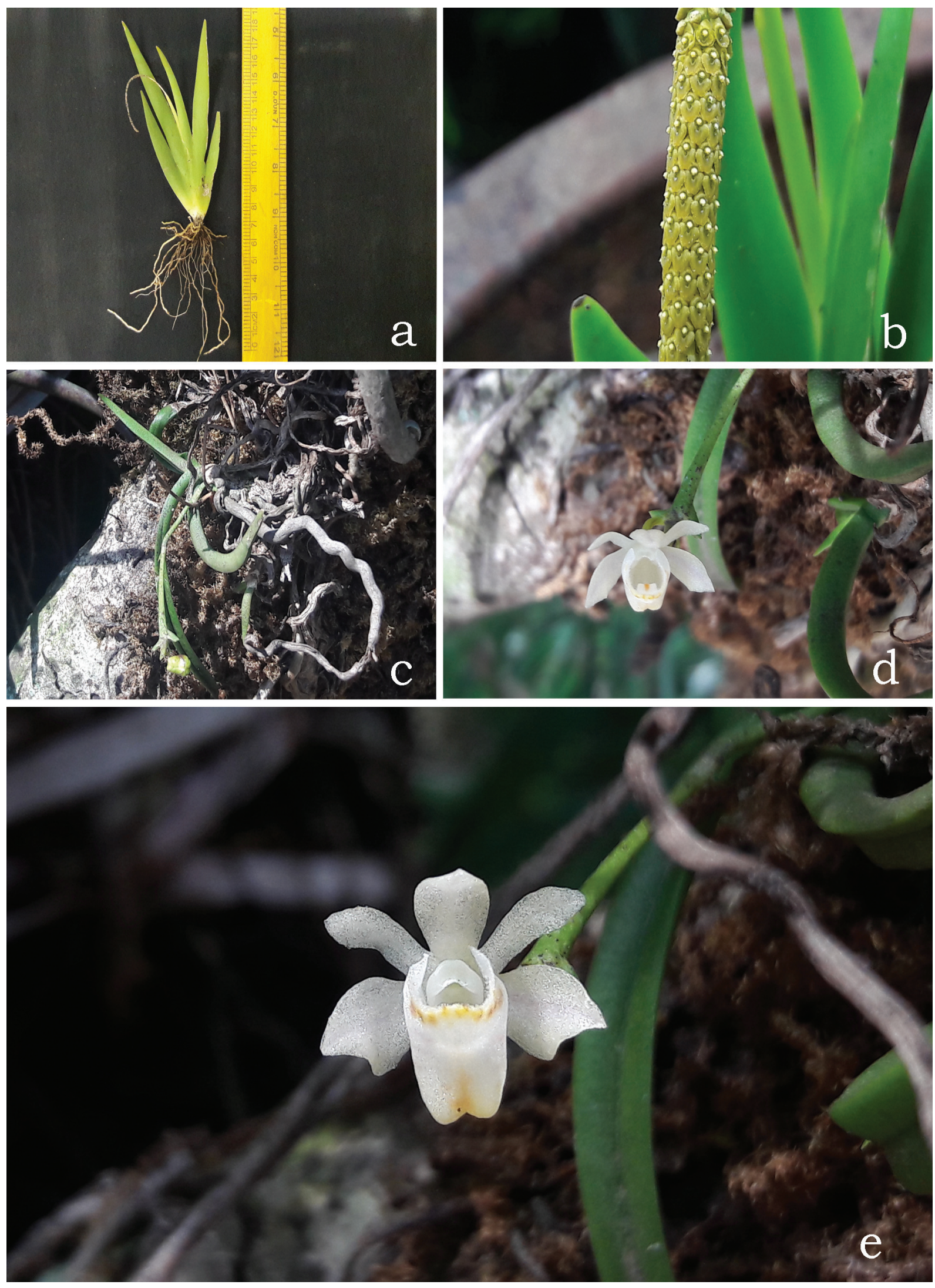

Fig. 1. a \& b. Oberonia maxima C.S.P.Parish ex Hook.f. a. Habit; b. Inflorescence. c-e. Thrixspermum formosanum (Hayata) Schltr. c. Habit; d. Flower: lip showing callus; e. Flower - Closer view. 
laciniate towards apex, gland-dotted, greenish. Sepals reflexed, gland-dotted, imbricate, glabrous; dorsal sepal c. $1.4 \times 1.1 \mathrm{~mm}$, ovate, obtuse at apex, entire, erect when young; lateral sepals $c .1 .4 \times 1.1$ $\mathrm{mm}$, ovate, acute at apex, irregularly dentate at apices. Lateral petals $c .1 .3 \times 1.0 \mathrm{~mm}$, narrowly ovate, obtuse at apex, irregularly dentate at apices; lip c. $1.5 \times 1.9 \mathrm{~mm}$, broader than long, orbicular, concave, crenulate at margins, gland-dotted; column c. $1 \mathrm{~mm}$ long, pale yellow; pollinia 2, obovate. Capsules 4-5 mm long, ellipsoid, shortly stalked, ridged.

Flowering \& fruiting: September-November.

Ecology: This species was found growing on moss covered tree trunks in evergreen forests, in association with Oberonia verticillata Wight and O. wightiana Lindl., at elevations between 2000 and $2300 \mathrm{~m}$.

Distribution: India, Borneo, Laos, Myanmar, Sabah, South and SE China, Thailand and Vietnam.

Specimen examined: INDIA, Tamil Nadu, Nilgiri Biosphere Reserve, Mukurthi National Park, $11^{\circ} 15^{\prime}$ 31" N, 76030' 14" E $\pm 2241.6 \mathrm{~m}, 21.09 .2018, S$. Kaliamoorthy \& T.S. Saravanan 109649 (MH).

Conservation status: Less than 100 mature individuals were noticed during the field survey at Mukurthi National Park. Extensive field study in similar habitats in the adjacent localities is required to determine its exact IUCN threat status. Live specimens brought from the study area are being multiplied and maintained as germplasm collections at NOEG, BSI, SRC, Yercaud.

Thrixspermum formosanum (Hayata) Schltr., Repert. Spec. Nov. Regni Veg. Beih. 4: 273. 1919; H.J.Su, Fl. Taiwan 5: 1049. 1978; S.C.Chen \& J.J. Wood, Fl. China 25: 468. 2009; K.Gogoi \& R.Yonzone, McAllen Int. Orchid Soc. J. 16(1): 12. 2015.

Fig. 1c-e

Small, epiphytic herbs, 6-9 cm tall. Roots 3.4-5.3 $\mathrm{cm}$ length, $0.2-0.3 \mathrm{~cm}$ in diam., radicosus, white. Stems ascending, 1.3-2.2 × 0.4-0.6 cm. Leaves distichous, $1.3-4.6 \times 0.3-0.5 \mathrm{~cm}$, linear-lanceolate, acute, jointed at base, thick and fleshy, midrib grooved ventrally, convex dorsally, green, spotted with purple. Inflorescence axillary racemes at lower nodes, shorter than leaves; peduncle $2.5 \mathrm{~cm}$ long, suberect, slender, straight, with 2 sterile bracts; rachis short, $0.3 \mathrm{~cm}$ long, green, thickened, few flowered, opening successively, lasting half a day; floral bracts few, c. $0.1 \mathrm{~cm}$ long, broadly ovate-triangular, acute at apex, green, persistent on rachis. Flowers c. $1.3 \times$ $1.0 \mathrm{~cm}$, fragrant; pedicel plus ovary $0.5 \mathrm{~cm}$ long, greenish brown. Sepals subsimilar, gland dotted; dorsal sepal 0.5-0.6 × 0.2-0.3 cm, oblong, obtuse; lateral sepals $0.5-0.6 \times 0.2-0.4 \mathrm{~cm}$, obliquely obovate, obtuse at apex. Lateral petals $0.6-0.7 \times$ 0.1-0.2 cm, falcate-oblong, obtuse; lip white with brown stripes, saccate at base, 3-lobed; lateral lobes erect, $0.2-0.3 \times 0.3-0.4 \mathrm{~cm}$, subovate, obtuse; midlobe inconspicuous, disc pubescent; callus running from base to mid-lobe, apex of callus protruding, notched below mid-lobe; sac $0.4-0.6 \mathrm{~cm}$ long, cylindric, shallowly bilobed at base; column short, c. $0.15 \mathrm{~cm}$ long, foot c. $0.2 \mathrm{~cm}$ long, white. Anther cap sub-orbicular, white; pollinia 4, in two nearly equal pairs, attached by a short and broad stipe to a solitary viscidium. Capsules $4.5-7.3 \times 0.3-0.4 \mathrm{~cm}$, linear, cylindrical.

Flowering \& fruiting: April-May.

Habitat: A rare epiphytic orchid found growing on moss covered tree trunks in evergreen forest at elevations between 1950 and $2300 \mathrm{~m}$.

Distribution: India, China, Taiwan and Vietnam.

Specimen examined: INDIA, Tamil Nadu, Nilgiri Biosphere Reserve, Mukurthi National Park, $11^{\circ} 15^{\prime}$ $31^{\prime \prime} \mathrm{N}, 76^{\circ} 30^{\prime} 14^{\prime \prime} \mathrm{E} \pm 2236 \mathrm{~m}, 18.11 .2018, \mathrm{~S}$. Kaliamoorthy \& T.S. Saravanan 109641 (MH).

Conservation status: Less than 25 mature individuals were noticed during the field survey at Mukurthi National Park. Extensive field study in similar habitats in the adjacent localities is required to determine its exact IUCN threat status. Live specimens brought from the study area are being multiplied and maintained as germplasm collections at NOEG, BSI, SRC, Yercaud. 


\section{Acknowledgements}

The authors are thankful to the Director, BSI, Kolkata and Scientist 'E' \& Head of Office, BSI, SRC, Coimbatore, for facilities and encouragement; the Principal Chief Conservator of Forests, Chennai and Forest Department authorities of Mukurthi National Park, Nilgiri Biosphere Reserve, Tamil Nadu, for granting permission and guidance in the field.

\section{Literature Cited}

ANSARI R. \& N.P. BALAKRISHNAN 1990. A revision of the Indian species of Oberonia (Orchidaceae). Orchid Monographs 4: 1-82.

CHEN X. \& J.J. WOOD 2009. Thrixspermum. In: WU Z.Y., RAVEN P.H. \& D.Y. HONG (eds.), Flora of China. Volume 25. Orchidaceae. Science Press, Beijing and Missouri Botanical Garden Press, St. Louis. pp. 466-470.

CHOWLU K. \& K.S. RAB 2017. Oberonia jhae: a new species of orchid from Arunachal Pradesh, India. Bangladesh Journal of Plant Taxonomy 24(1): 49-52.

CHOWLU K., NANDA Y., RAO A.N., ANGELA N., BISHWAJIT H. \& G. AKIMPOU 2015. Oberonia manipurensis sp. nov. (Orchidaceae) from Manipur, India. Nordic Journal of Botany 33: 42-44.

DEORI N.C. \& P.K. HAJRA 1975. Oberonia maxima Hook.f. An interesting Orchid from Kameng District, Arunachal Pradesh. Bulletin of Botanical Survey of India 17(1-4): 170-171.

GEIGER D. L. 2016. Studies in Oberonia 1: Oberonia maxima and its new synonyms. Orchids 85(5): 390-395.

GOGOI K. \& R. YONZONE 2015. Thrixspermum formosanum (Hayata) Schltr. (Orchidaceae) from Karbi Anglong (Assam): A new record for India. McAllen International Orchid Society Journal 16(1): 11-16.

GOGOI K. 2011. Thrixspermum acuminatissimum (Blume) Rchb.f. (Orchidaceae) - a recollection for India. Pleione 5(2): 334-336.
GOVAERTS R., BERNET P., KRATOCHVIL K., GERLACH G., CARR G., ALRICH P., PRIDGEON A.M., PFAHL J., CAMPACCI M.A., HOLLAND D. BAPTISTA TIGGES H., SHAW J., CRIBB P., GEORGE A., KREUZ K. \& J.J. WOOD 2019. World Checklist of Orchidaceae. Royal Botanic Gardens, Kew. http://apps.kew.org/wcsp/home.do [Accessed on 27.06.2019].

HYNNIEWTA T.M., KATAKI S.K. \& B.M. WADHWA 2000. Orchids of Nagaland. Botanical Survey of India, Kolkata. pp. 1-306.

KUMAR C.S. \& K.S. MANILAL 1994. A catalogue of Indian Orchids. Bishen Singh Mahendra Pal Singh, Dehra Dun. pp. 1-162.

KUMAR C.S. \& P.C.S. KUMAR 2005. An Orchid Digest of Manipur, Northeastern India. Rheedea 15(1): 50-51.

LIU T.S. \& H.J. SU 1978. Orchidaceae. In: LI H.L. et al. (eds.), Flora of Taiwan. Volume 5. National Taiwan University, Taipei. pp. $859-1137$.

MAINA V., RAO P.S.N. \& B.K. SINHA 1998. A new record of Thrixspermum merguense (Hook.f.) Kuntze (Orchidaceae) from Nicobar Islands. Journal of the Bombay Natural History Society 95(2): 375-376.

MAO A.A., RAO A.N., BHAUMIK M. \& O. APANG 2011. Thrixspermum saruwatarii (Hayata) Schltr. (Orchidaceae) - a new record to India from Arunachal Pradesh. Bulletin of Arunachal Forest Research 26(1\&2): 68-70.

MISRA S. 2007. Orchids of India - a glimpse. Bishen Singh Mahendra Pal Singh, Dehra Dun. pp. 1-402.

NARAYANAN M.K.R., MANUDEV K.M., SUJANAPAL P., KUMAR N.A., SIVADASAN M. \& A.H. ALFARHAN 2010. Oberonia swaminathanii sp. nov. (Orchidaceae) from Kerala, India. Nordic Journal of Botany 28(6): 713-715.

PRASAD K., PRABHUKUMAR K.M. \& P. SUDHESHNA 2018. A new species of Oberonia (Orchidaceae) from Western Ghats of Kerala, India. Nordic Journal of Botany 36(5): e01797.1-4.

THE PLANT LIST 2013. Version 1.1. Published on the Internet; http://www.theplantlist.org/ (accessed 27.06.2019). 